Nebel, S., J. Casey, M.-A. Cyr, K. J. Kardynal, E. A. Krebs, E. F. Purves, M. Bélisle, R. M. Brigham, E. C. Knight, C. Morrissey, and R. G. Clark. 2020. Falling through the policy cracks: implementing a roadmap to conserve aerial insectivores in North America. Avian Conservation and Ecology 15(1):23. https://doi.org/10.5751/ACE-01618-150123

Copyright (C) 2020 by the author(s). Published here under license by the Resilience Alliance.

Guest Editorial

\title{
Falling through the policy cracks: implementing a roadmap to conserve aerial insectivores in North America
}

Silke Nebel ${ }^{1}$, James Casey ${ }^{1}$, Marc-André Cyr ${ }^{2}$, Kevin J. Kardynal ${ }^{3}$, Elizabeth A. Krebs ${ }^{4}$, Elisabeth F. Purves ${ }^{1}$, Marc Bélisle $^{5,6}$, R. Mark Brigham ${ }^{7}$, Elly C. Knight ${ }^{8}$, Christy Morrissey ${ }^{9}$ and Robert G. Clark ${ }^{4,9}$

${ }^{1}$ Birds Canada, ${ }^{2}$ Environment and Climate Change Canada, Canadian Wildife Service, ${ }^{3}$ Environment and Climate Change Canada, Science and Technology Branch, ${ }^{4}$ Environment and Climate Change Canada, Wildlife Research Division, Wildlife and Landscape Science Directorate, ${ }^{5}$ Département de Biologie, Université de Sherbrooke, Canada, ${ }^{6}$ Centre d'Étude de la Forêt (CEF), ${ }^{7}$ Department of Biology, University of Regina, ${ }^{8}$ University of Alberta, ${ }^{9}$ Department of Biology, University of Saskatchewan

\section{Laissés pour compte par les politiques: Mise en oeuvre d'une feuille de route pour la conservation des insectivores aériens en Amérique du Nord}

Key Words: aerial insectivores; agriculture; climate change; forestry; insects; land use change; pesticides; policy gaps Mots clés: agriculture; changement climatique; changement d'affectation des terres; failles entre les politiques; foresterie; insectes; insectivores aériens; pesticides

The rapid spread and impact of the COVID-19 pandemic serves as a sharp reminder about how closely connected we are to each other and our environment. This means that policy decisions in one region of the world can produce severe repercussions in others. We have been forced to slow our pace of life, a change that has left many reflecting on how the modern economy shapes our lives and the natural world. The cumulative impact of past and recent policy decisions has produced widespread changes to North America's landscapes, its avifauna and its once-rich biodiversity. Swift and effective action across the globe to reduce the spread and impact of COVID-19 demonstrates the possibility for similarly rapid action to prevent declining bird populations from "falling through the cracks."

Monitoring trends in bird populations in agricultural and forested environments, i.e., working landscapes, provides an efficient way of tracking changes in ecosystem health (e.g., Gregory et al. 2005). Populations of avian aerial insectivores - birds that catch insects on the wing - have been declining for decades, inspiring early calls for conservation actions (Nebel et al. 2010). Recent population estimates reinforce the need to take concrete action: aerial insectivores have declined by an estimated 59\% since the 1970 s in Canada and $\sim 32 \%$ across North America (NABCI 2019, Rosenberg et al. 2019). This is the most severe decline of any group of birds, and translates into the loss of more than 160 million individuals across Canada and the United States. Nine of the 31 species of flycatchers, nightjars, swallows, and swifts are currently listed in Canada under the federal Species at Risk Act (https:// www.canada.ca/en/environment-climate-change/services/speciesrisk-public-registry.html). These stark trends in bird populations send a clear message about the health of our working landscapes. Also, immediate action is needed if we are to meet Canada's 2020 national biodiversity goal to maintain ecosystems that support biodiversity conservation outcomes (Goal A; https:// biodivcanada.chm-cbd.net/2020-biodiversity-goals-and-targets-canada).

Most impacts linked to cumulative effects of land use change and climate change

Aerial insectivores are subject to myriad anthropogenic stressors across their annual cycles but there is little evidence that a single factor explains the fluctuations observed among populations and species (Michel et al. 2016, Spiller and Dettmers 2019). The expansion of intensive agricultural and urban land use especially over the last 30 years has introduced a range of threats impacting both habitat quantity and quality. Direct impacts include habitat loss and degradation, particularly of wetlands, grasslands, and forests, and indirect impacts of stressors that reduce the quality of breeding, foraging, and roosting sites. Cumulatively, systemic use of pesticides has cascading effects across ecosystems, affecting avian health and food webs (e.g., Chagnon et al. 2015). A comprehensive review concludes, "the wide-scale use of these... chemicals is having widespread, chronic impacts upon global biodiversity and is likely to be having major negative effects on ecosystem services such as pollination that are vital to food security and sustainable development" (Bijleveld van Lexmond et al. 2015).

Collectively, these threats are intersected and often intensified by climate change, which can alter breeding distributions and disrupt the phenology of aerial insect availability (Imlay et al. 2018). Climate change can also increase the frequency and severity of extreme weather events, which reduce breeding success or increase the costs of migration. The ephemeral nature of flying insects could make both insects and their consumers particularly 
vulnerable to changes in climate variability and patterns. These large-scale threats likely interact, amplifying cumulative biological effects on migratory species such as the aerial insectivores.

Because aerial insects form a common trophic link across this guild, it is tempting to focus on the apparent catastrophic declines in global insect populations as the underlying cause for aerial insectivore declines (e.g., Hallmann et al. 2014, 2017). In Europe, declines in swallow abundance correlate with insect declines over a 20-year period suggesting that prey declines may, at least partially, be responsible for aerial insectivore declines (Møller 2019; also see Wagner 2020). Entomologists are debating the quality of evidence surrounding reported insect collapses (Didham et al. 2020, Montgomery et al. 2020), and the extent and severity of changes in insect populations worldwide (van Klink et al. 2020). Thus, a rigorous assessment of aerial insect communities in landscapes dominated by different land uses, approaches to sustaining or increasing insect populations, and possible causes of insect declines would be both timely and informative.

\section{A call to action: informing the development of a conservation road map}

The highly variable spatial extent and severity of declines among aerial insectivores suggest widespread, diverse environmental impacts can only be adequately addressed by a coordinated response to improve habitat and environmental quality, for birds and for people. We must find ways to more effectively engage stakeholders who influence land management policies at all levels from local to international and codevelop solutions. We recommend five initial steps toward an aerial insectivore conservation roadmap, beginning with immediate "no-regrets" actions all the way to long-term international policy initiatives (Table 1):

Step 1. Implement a range of attainable "no-regrets" actions that, regardless of their effects on aerial insectivore populations, are likely to have positive benefits for biodiversity. We should:

- develop targeted communication strategies to increase public awareness of the role of insects in ecosystems, and aerial insectivores as indicators of environmental health;

- support incentives to align agricultural production with ecological processes while reducing reliance on external agrochemical inputs;

- follow the principles of Integrated Pest Management to encourage the use of environmentally benign pesticides at small scales when required and to reduce prophylactic use of harmful pesticides at large scales, e.g., neonicotinoidtreated seeds of most crops (Furlan et al. 2018);

- take actions to reduce avian mortality at home and at work (https://www.birdscanada.org/you-can-help/top-6-ways-tohelp-birds/; Calvert 2012, Longcore and Smith 2013 review causes of avian mortality);

- reduce light pollution to lower losses of insects (Wagner 2020), and collision risk to birds and bats;

- expand green belts, parks, and urban forests to buffer effects of urbanization, and;
- protect "heritage" farms and structures, e.g., barns, in agricultural landscapes.

Step 2. Assess existing and proposed federal and provincial land management regulations, pinpoint "cracks," i.e., gaps (Fig. 1) in Canada's policy landscape, and suggest areas for improvement. Implement specific actions to:

- ensure that environmental policies of federal (Agriculture and Agri-Food Canada [AAFC], Natural Resources Canada, Health Canada, Environment and Climate Change Canada [ECCC]), provincial and municipal governments are aligned to produce cobenefits for people, aerial insectivores, and the environment;

- work with AAFC to implement a broad policy about beneficial insects like pollinators and parasitoids, recognizing that other insects and biota provide numerous benefits to producers and society;

- re-engage ECCC in the Federal, Provincial, Territorial (FPT) Committee on Pest Management and Pesticides to ensure that regulations and standards on pesticide use are informed by science, ensure healthy ecosystems, and align with conservation initiatives for avian and other wildlife populations, and;

- strengthen wetland protection and management across all Canadian jurisdictions to provide ecosystem services and cobenefits such as in carbon sequestration and flooddrought risk reduction. Current federal priorities in naturebased solutions could provide rationale and resources for this purpose. As the Canada Water Agency evolves, seek to ensure this agency implements countrywide actions to protect and restore wetlands in urban, agricultural, and forested environments.

Step 3. Codevelop and implement land management approaches with partners in agriculture and forestry to safeguard appropriate habitat (e.g., native grasslands), enhance landscape heterogeneity, and to convey the cobenefits of sustainable environmental management. Specifically,

- provide clear guidance on environmentally functional landscape conditions (e.g., Kleijn et al. 2019), along with compatible applications of pesticides to reduce harmful substances in insect habitats, and;

- explore and create (i) financial incentives and (ii) market opportunities for certification programs that recognize producers who adopt biodiversity-friendly practices, e.g., agro-ecological schemes.

Step 4. Canada currently lacks legislative provisions for insects, e.g., pollinators, parasitoids, or other species, at the federal and provincial levels (Tang et al. 2007). Germany is building a program for insect protection that includes a legally binding insect protection act (https://www.bmu.de/en/download/action-programmefor-insect-protection-1/), financial investments in protection of insects and their habitats, and public outreach. Recently, > 50 scientists from across the globe called for a roadmap to insect protection and recovery (Harvey et al. 2020), suggesting an opportunity to:

- consult widely with policy/science experts to explore solutions to common challenges in insect conservation; 
Avian Conservation and Ecology 15(1): 23

http://www.ace-eco.org/vol15/iss1/art23/

Table 1. Achievable steps to remediate "cracks," i.e., gaps, in Canada's policy landscape (Fig. 1), and improve conditions for human health, the environment, and aerial insectivores.

\begin{tabular}{ll}
\hline \hline Steps & Objectives \\
\hline 1. "No-regret" actions & $\begin{array}{l}\text { Public understands role of birds and insects } \\
\text { as indicators of environmental health. }\end{array}$
\end{tabular}

Agro-ecological practices and integrated pest management restored, pesticide use reduced.

Avian mortality is reduced by individuals at home and at work.

Light pollution is reduced to conserve energy; save insects, birds, and bats.

Urban habitat is improved with green belts, parks, and urban forests.

Heritage farms and structures are protected.

2. Policy assessments

Land management, agricultural and related policies align, produce cobenefits for people and the environment.

Agriculture and Agri-Foods Canada develops policy on beneficial insects. Environment and Climate Change Canada is represented on the Pest Management Regulatory Agency's Federal, Provincial, Territorial (FPT) Committee on Pest Management and Pesticides.

Wetland protection strengthens across all Canadian jurisdictions.

Water management improved for human and biodiversity benefits.

3. Codevelop opportunities Conserve habitat, enhance heterogeneity. in agriculture and forestry

Ecologically functional landscape conditions defined, use of harmful substances reduced.

Develop financial incentives and marketdriven certification programs that recognize biodiversity-friendly practices.

4. Insect conservation

Support insect conservation initiatives. Seek solutions to common conservation challenges with EU policy/science experts.

5. Enhance environmental cooperation and communication
Table 1. Mesures réalisables pour remédier aux lacunes dans le cadre stratégique du Canada (Fig. 1) et améliorer les conditions de la santé humaine, de l'environnement, et des insectivores aériens.

\begin{tabular}{ll}
\hline \hline Mesures & Objectifs \\
\hline 1. Mesures «sans regret» & $\begin{array}{l}\text { Le public comprend le rôle des oiseaux et des } \\
\text { insectes en tant qu'indicateurs de la santé de }\end{array}$
\end{tabular}
l'environnement.

Les pratiques agroécologiques et la lutte intégrée contre les ravageurs sont rétablies, l'utilisation des pesticides est réduite. La mortalité aviaire est réduite par les particuliers, à la maison et au travail. La pollution lumineuse est réduite pour préserver l'énergie, les insectes, les oiseaux, et les chauves-souris.

L'habitat urbain est enrichi par des ceintures vertes, des parcs, et des forêts urbaines

Les fermes et les structures patrimoniales sont protégées

2. Évaluation des Les politiques en matière de gestion des politiques terres, d'agriculture et connexes sont harmonisées et produisent des avantages connexes pour les personnes et l'environnement.

Agriculture et Agroalimentaire Canada élabore une politique sur les insectes utiles. Environnement et Changement climatique Canada est représenté au sein du Comité fédéral, provincial et territorial (FPT) sur la lutte antiparasitaire et les pesticides de l'Agence de réglementation de la lutte antiparasitaire.

La protection des milieux humides est renforcée dans toutes les provinces et tous les territoires du Canada.

Amélioration de la gestion de l'eau pour le bénéfice des humains et de la biodiversité.

3. Évaluation conjointe des Conserver les habitats, renforcer possibilités dans les l'hétérogénéité. domaines agricole et forestier

Définir les conditions des paysages écologiquement fonctionnels, réduction de l'utilisation de substances nocives.

Créer des incitatifs financiers et des programmes de certification axés sur le marché qui reconnaissent les pratiques respectueuses de la biodiversité.

4. Conservation des insectes

Soutenir la conservation des insectes. Cherchez des solutions aux problèmes communs de conservation avec les experts en politiques et en science de l'Union européenne.

5. Renforcer la coopération Les normes canadiennes ou des normes plus et la communication en matière d'environnement élevées sont maintenues dans les accords de libre-échange et autres accords sur la production de biens.

Les politiques en matière de conservation des habitats et d'agriculture, par exemple sur les pesticides, ont été améliorées dans l'hémisphère occidental.

Conservation des oiseaux migrateurs néarctiques-néotropicaux en Amérique latine. 
Fig. 1. Infographic of key threats encountered by aerial insectivores throughout their annual life cycles. Many of these threats result from the cumulative effects of land use practices that reflect policy shortfalls or gaps; we refer to these as "cracks." Updated policies in agriculture, forestry, and urban planning should aim to seal these cracks.
Fig. 1. Infographie des principales menaces rencontrées par les insectivores aériens tout au long de leur cycle de vie annuel. Nombre de ces menaces résultent des effets cumulés des pratiques d'utilisation des terres qui reflètent les lacunes des politiques ou les failles entre celles-ci. Des politiques actualisées dans les secteurs de l'agriculture, de la foresterie, et de l'aménagement urbain devraient viser à colmater ces failles.

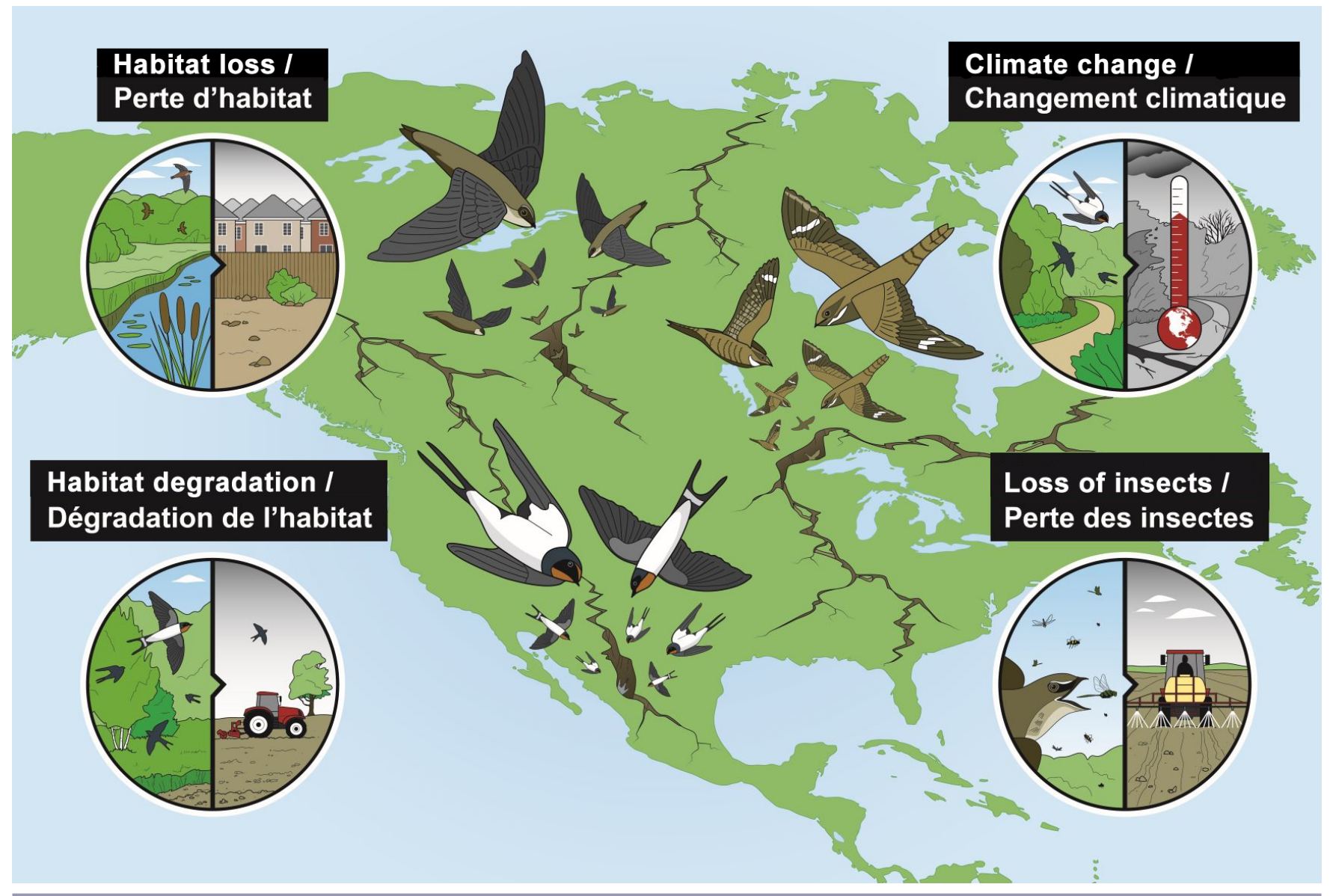

- develop and implement a Canadian roadmap to insect protection.

Step 5. Take steps to enhance environmental cooperation and establish a dialogue about the cobenefits for aerial insectivores among governments in the Western Hemisphere and increase dialogue with experts of the Afro-Palearctic system where aerial insectivores are undergoing similar declines. Build on momentum created by the Commission for Environmental Cooperation (CEC), North American Waterfowl Management Plan (NAWMP), Partners in Flight (PIF), and other initiatives, to:

- develop opportunities for enhanced environmental cooperation and communication, and conservation action, in all regions of North America and in key countries in Central and South America where most aerial insectivores spend the nonbreeding period;
- enhance and refine habitat conservation/agriculture, e.g., pesticide, policies;

- ensure free trade and other international agreements require production systems that meet Canadian standards, e.g., agriculture, forestry, and mining;

- improve multilateral engagement and policy/international government collaborations, e.g., agreements with Latin American countries for conservation of habitats for "shared" migratory bird species, and increase the profile of aerial insectivores and other Nearctic-Neotropical migrant birds and their conservation in Latin America, and;

- engage EU policy/science experts to explore solutions to common challenges in migratory bird conservation.

Take action, now! We have recommended several steps to implement a roadmap to aerial insectivore conservation. Many 
of these actions would improve environmental quality for all biodiversity. They would also reduce socioeconomic risks to industry and Canadians, create more resilient and sustainable ecosystems within working landscapes, and contribute to meeting Canada's national biodiversity goals.

New research will continue to inform our understanding of aerial insectivores and their aerial insect prey populations, their roles as indicators of ecosystem health, and the factors driving declines. However, similar efforts are urgently needed to implement transformative policy reforms and innovations, particularly in the agriculture and forestry sectors, including those that identify a sustainable pace of development. A key challenge is to mobilize resources and implement practical solutions that will benefit people and the environment. The response by Canadians to the COVID-19 pandemic amply demonstrates that rapid changes are possible when we work together to surmount challenges.

La propagation rapide et l'impact de la pandémie de COVID-19 nous rappellent avec force à quel point nous sommes étroitement liés les uns aux autres et à notre environnement. Cela signifie que les décisions politiques prises dans une région peuvent avoir de graves répercussions dans d'autres parties du monde. Nous avons été contraints de ralentir notre rythme de vie, un changement qui a amené de nombreuses personnes à réfléchir à la manière dont l'économie moderne façonne nos vies et le monde naturel. L'effet cumulé des décisions politiques passées et récentes a entraîné des changements importants dans les paysages de l'Amérique du Nord, son avifaune et sa biodiversité autrefois riche. Les mesures rapides et efficaces déployées dans le monde entier pour réduire la propagation et l'impact de la COVID-19 montrent qu'une action tout aussi rapide est possible pour éviter que les populations d'oiseaux en déclin ne passent entre les mailles du filet.

La surveillance des tendances des populations d'oiseaux dans les milieux agricoles et forestiers (c.-à-d. les paysages fonctionnels) offre un moyen efficace de suivre les changements dans la santé des écosystèmes (p. ex. Gregory et al. 2005). Les populations d'oiseaux insectivores aériens (oiseaux qui attrapent des insectes au vol) sont en déclin depuis des décennies, ce qui a inspiré les premiers appels à des mesures de conservation (Nebel et al. 2010). Des estimations récentes des populations soulignent la nécessité de prendre des mesures concrètes : on estime que le nombre d'insectivores aériens a diminué de $59 \%$ depuis les années 1970 au Canada et d'environ 32\% en Amérique du Nord (NABCI 2019, Rosenberg et al. 2019). Il s'agit du déclin le plus important parmi les groupes d'oiseaux, qui se traduit par la perte de plus de 160 millions d'individus au Canada et aux États-Unis. Neuf des 31 espèces de moucherolles, d'engoulevents, d'hirondelles et de martinets sont actuellement inscrites au Canada sur la liste de la Loi sur les espèces en péril (https://www.canada.ca/fr/ environnement-changement-climatique/services/registre-public-especesperil.html). Ces tendances frappantes dans les populations d'oiseaux envoient un message clair sur la santé de nos paysages fonctionnels. De plus, des mesures immédiates sont nécessaires si nous voulons atteindre l'objectif national de biodiversité du Canada pour 2020, qui consiste à maintenir des écosystèmes qui soutiennent les résultats en matière de conservation de la biodiversité (objectif A; https://biodivcanada.chm-cbd.net/fr/ buts-et-objectifs-canadiens-pour-la-biodiversite-dici-2020) .

\section{Impacts principalement liés aux effets cumulatifs du changement} d'affectation des terres et du changement climatique

Les insectivores aériens sont soumis à une myriade de facteurs de stress anthropiques au cours de leurs cycles annuels, mais rien ne semble indiquer qu'un seul facteur explique les fluctuations observées parmi les populations et les espèces (Michel et al. 2016, Spiller et Dettmers 2019). L'expansion de l'utilisation intensive des terres agricoles et urbaines, en particulier au cours des 30 dernières années, a introduit une série de menaces qui ont un impact à la fois sur la quantité et la qualité des habitats. Les impacts directs comprennent la perte et la dégradation des habitats, en particulier les zones humides, les prairies, et les forêts, et les impacts indirects des facteurs de stress qui réduisent la qualité des sites de reproduction, d'alimentation, et de repos. Cumulativement, l'utilisation systémique de pesticides a des effets en cascade sur les écosystèmes, qui touchent la santé des oiseaux et les chaînes alimentaires (voir Chagnon et al. 2015). Une étude exhaustive conclut que « l'utilisation à grande échelle de ces produits chimiques a des effets chroniques et étendus sur la biodiversité mondiale et aura probablement des effets négatifs majeurs sur les services écosystémiques tels que la pollinisation, qui sont vitaux pour la sécurité alimentaire et le développement durable » (traduction de Bijleveld van Lexmond et al. 2015).

Collectivement, ces menaces se recoupent et sont souvent intensifiées par le changement climatique, qui peut modifier la répartition des aires de reproduction et perturber la phénologie de la disponibilité des insectes volants (Imlay et al. 2018). Le changement climatique peut également accroître la fréquence et la gravité des phénomènes météorologiques extrêmes qui réduisent le succès de la reproduction ou augmentent les coûts de la migration. La nature éphémère des insectes volants pourrait rendre les insectes et leurs consommateurs particulièrement vulnérables aux changements de la variabilité et des régimes climatiques. Ces menaces à grande échelle interagissent probablement, amplifiant les effets biologiques cumulés sur les espèces migratrices telles que les insectivores aériens.

Puisque les insectes aériens représentent un lien trophique commun à travers cette guilde, il est tentant de se concentrer sur le déclin apparemment catastrophique des populations mondiales d'insectes comme cause sous-jacente du déclin des insectivores aériens (voir Hallmann et al. 2014, 2017). En Europe, la diminution de l'abondance des hirondelles est corrélée avec le déclin des insectes sur une période de $20 \mathrm{ans}$, ce qui donne à croire que le déclin des proies pourrait, au moins partiellement, être responsable du déclin des insectivores aériens (Møller 2019; voir aussi Wagner 2020). Les entomologistes débattent de la qualité des preuves entourant les effondrements d'insectes rapportés (Didham et al. 2020, Montgomery et al. 2020), ainsi que de l'étendue et de la gravité des changements dans les populations d'insectes à travers le monde (van Klink et al. 2020). Il serait à la fois opportun et instructif de mener une évaluation rigoureuse des communautés d'insectes aériens dans les paysages dominés par différentes utilisations des terres, des approches pour maintenir ou augmenter les populations d'insectes, et des causes possibles du déclin des insectes. 


\section{Un appel à l'action: contribuer à l'élaboration d'une feuille de route pour la conservation}

L'étendue spatiale et la gravité très variables des déclins des insectivores aériens indiquent que les impacts environnementaux étendus et variés ne peuvent être traités de manière adéquate que par une réponse coordonnée visant à améliorer la qualité de l'habitat et de l'environnement, pour les oiseaux et pour les humains. Nous devons trouver des moyens de mobiliser de manière plus efficace les parties prenantes qui influent sur les politiques de gestion des terres à tous les niveaux, du local à l'international, et élaborer des solutions de manière conjointe. Nous recommandons cinq étapes initiales vers une feuille de route pour la conservation des insectivores aériens, en commençant par des mesures immédiates « sans regret » jusqu'aux initiatives politiques internationales à long terme (tableau 1).

Étape 1. Mettre en œuvre une série de mesures «sans regret » réalisables qui, indépendamment de leurs effets sur les populations d'insectivores aériens, sont susceptibles d'avoir des retombées positives pour la biodiversité. Nous devrions :

- mettre au point des stratégies de communication ciblées pour sensibiliser le public au rôle des insectes dans les écosystèmes et des insectivores aériens en tant qu'indicateurs de la santé environnementale;

- soutenir des incitatifs visant une production agricole respectueuse des processus écologiques tout en réduisant la dépendance à l'égard des intrants agrochimiques externes;

- suivre les principes de la lutte antiparasitaire intégrée pour encourager l'utilisation, lorsque nécessaires, de pesticides respectueux de l'environnement à petite échelle et pour réduire l'utilisation prophylactique de pesticides nocifs à grande échelle, par exemple, les semences traitées aux néonicotinoïdes de la plupart des cultures (Furlan et al. 2018);

- Agir afin de réduire la mortalité aviaire à la maison et au travail (https://www.oiseauxcanada.org/vous-pouvez-aider/ les-6-meilleures-facons-daider-les-oiseaux/; Calvert 2012, examen des causes de la mortalité aviaire par Longcore et Smith 2013);

- réduire la pollution lumineuse pour diminuer les pertes d'insectes (Wagner 2020) et les risques de collision pour les oiseaux et les chauves-souris;

- étendre les ceintures vertes, les parcs, et les forêts urbaines pour atténuer les effets de l'urbanisation, et ;

- protéger les fermes et les structures patrimoniales, telles que les granges, dans les paysages agricoles.

Étape 2. Évaluer les règlements fédéraux et provinciaux, existants et proposés, en matière de gestion des terres, identifier les failles dans le cadre stratégique canadien (Fig. 1) et suggérer des améliorations. Mettre en œuvre des mesures spécifiques pour :

- veiller à ce que les politiques environnementales des gouvernements fédéral (Agriculture et Agroalimentaire Canada [AAC], Ressources naturelles Canada, Santé Canada et Environnement et Changement climatique Canada [ECCC]), provinciaux et municipaux soient harmonisées de manière à ce qu'elles produisent des avantages conjoints pour les personnes, les insectivores aériences, et l'environnement;

- collaborer avec AAC pour mettre en œuvre une politique générale concernant les insectes bénéfiques comme les pollinisateurs et les parasitoïdes, en reconnaissant que les autres insectes et biotes offrent de nombreux avantages aux producteurs et à la société;

- réintégrer ECCC dans le Comité fédéral, provincial et territorial (FPT) sur la lutte antiparasitaire et les pesticides afin de s'assurer que les règlements et les normes sur l'utilisation des pesticides sont éclairés par la science, favorisent des écosystèmes sains, et sont en cohérence avec les initiatives de conservation visant les populations aviaires et autres espèces sauvages;

- renforcer la protection et la gestion des milieux humides dans toutes les provinces et tous les territoires du Canada afin d'obtenir des services écosystémiques et des avantages connexes tels que la séquestration du carbone et la réduction des risques d'inondation et de sécheresse. Les priorités fédérales actuelles en matière de solutions basées sur la nature pourraient fournir une justification et des ressources à cette fin. À mesure que l'Agence canadienne de l'eau évoluera, veiller à ce que cette agence mette en œuvre des mesures à l'échelle nationale pour protéger et restaurer les zones humides dans les milieux urbains, agricoles, et forestiers.

Étape 3. Élaborer et mettre en œuvre des approches de gestion des terres avec des partenaires des secteurs agricole et forestier afin de sauvegarder les habitats appropriés (tels que les prairies indigènes), d'accroître l'hétérogénéité du paysage, et de faire valoir les avantages connexes de la gestion durable de l'environnement. Plus précisément :

- fournir des orientations claires sur les conditions environnementales des paysages fonctionnels (voir Kleijn et al. 2019), ainsi que sur les applications compatibles de pesticides pour réduire les substances nocives dans les habitats des insectes;

- évaluer et créer (i) des incitatifs financiers et (ii) des débouchés commerciaux pour les programmes de certification qui reconnaissent les producteurs qui adoptent des pratiques respectueuses de la biodiversité, par exemple, les programmes agro-écologiques.

Étape 4. Le Canada ne dispose pas actuellement de dispositions législatives concernant les insectes, par exemple, les pollinisateurs, les parasitoïdes, ou autres espèces, aux niveaux fédéral et provincial (Tang et al. 2007). L'Allemagne met en place un programme de protection des insectes qui comprend une loi juridiquement contraignante sur la protection des insectes (https://www.bmu.de/en/download/action-programme-for-insectprotection-1/), des investissements financiers dans la protection des insectes et de leurs habitats, et la sensibilisation du public. Récemment, plus de 50 scientifiques du monde entier ont demandé une feuille de route pour la protection des insectes et leur rétablissement (Harvey et al. 2020), ce qui démontre une occasion : 
- de consulter les experts en politiques et en sciences afin d'explorer des solutions aux défis communs en matière de conservation des insectes;

- d'élaborer et de mettre en œuvre une feuille de route pour la protection des insectes au Canada.

Étape 5. Prendre des mesures pour renforcer la coopération environnementale et établir un dialogue sur les avantages connexes des insectivores aériens entre les gouvernements de l'hémisphère occidental, et intensifier le dialogue avec les experts $\mathrm{du}$ système afro-paléarctique où les insectivores aériens connaissent un déclin similaire. Tirer parti de l'élan créé par la Commission de coopération environnementale (CCE), le Plan nord-américain de gestion de la sauvagine (PNAGS), Partenaires d'envol (PIF), et d'autres initiatives, pour :

- développer des opportunités de coopération et de communication environnementales renforcées, et des mesures de conservation, dans toutes les régions d'Amérique $\mathrm{du}$ Nord et dans les pays d'Amérique centrale et du Sud où la plupart des insectivores aériens passent la période internuptiale;

- améliorer et affiner les politiques en matière de conservation des habitats et d'agriculture, par exemple, les pesticides;

- faire en sorte que les accords de libre-échange et autres accords internationaux exigent que les biens produits soient conformes aux normes canadiennes, dans des secteurs tels que l'agriculture, la foresterie, et l'exploitation minière ;

- améliorer les accords multilatéraux et la collaborations des gouvernements sur les politiques internationales, par exemple, les accords avec les pays d'Amérique latine pour la conservation des habitats des espèces « partagées » d'oiseaux migrateurs, et accroître la visibilité des insectivores aériens et autres oiseaux migrateurs néarctiques-néotropicaux et leur conservation en Amérique latine, et ;

- faire appel à des experts en politiques et en sciences de l'Union européenne pour explorer des solutions aux défis communs en matière de conservation des oiseaux migrateurs.

Agissez, maintenant ! Nous avons recommandé plusieurs étapes pour la mise en œuvre d'une feuille de route pour la conservation des insectivores aériens. Nombre de ces mesures permettraient d'améliorer la qualité de l'environnement pour l'ensemble de la biodiversité. Ces mesures permettraient également de réduire les risques socio-économiques pour l'industrie et les Canadiens, de créer des écosystèmes plus résistants et plus durables au sein des paysages fonctionnels, et de contribuer à la réalisation des objectifs nationaux du Canada en matière de biodiversité.

De nouvelles recherches continueront d'éclairer notre compréhension des populations d'insectivores aériens et de leurs proies, de leur rôle en tant qu'indicateurs de la santé des écosystèmes et des facteurs à l'origine de leurs déclins. Toutefois, il est urgent de déployer des efforts similaires pour mettre en œuvre des réformes et des innovations politiques, notamment dans les secteurs agricole et forestier, permettant un développement durable. L'un des principaux défis consiste à mobiliser des ressources et à mettre en œuvre des solutions pratiques qui seront bénéfiques pour la société et l'environnement. La réaction des Canadiens à la pandémie de COVID-19 démontre amplement que des changements rapides sont possibles lorsque nous travaillons ensemble pour surmonter les difficultés.

Responses to this article can be read online at: http://www.ace-eco.org/issues/responses.php/1618

\begin{abstract}
Acknowledgments:
Conservation practitioners and scientists met in Saskatoon in March 2020 to discuss and prioritize research and policy needs and conservation actions for aerial insectivores. Motivated by these discussions, a policy working group was formed to focus on immediate actions to support aerial insectivore conservation. This editorial is an outcome of the workshop and those discussions. We sincerely appreciate ideas and constructive feedback from the Saskatoon Workshop participants. We thank Keith Hobson for the opportunity to prepare this editorial, and appreciate the helpful comments by Keith Hobson and Carolyn Callaghan.

Remerciements Des praticiens et des scientifiques de la conservation se sont réunis à Saskatoon en mars 2020 pour discuter et prioriser les besoins en matière de recherche et de politique ainsi que les mesures de conservation relatives aux insectivores aériens. Sur la base de ces discussions, un groupe de travail sur les politiques a été formé pour se concentrer sur les actions immédiates visant à soutenir la conservation des insectivores aériens. Cet éditorial est un résultat de l'atelier et de ces discussions. Nous apprécions sincèrement les idées et les commentaires constructifs des participants de l'atelier de Saskatoon. Nous remercions Keith Hobson de nous avoir donné l'occasion de préparer cet éditorial, et apprécions les commentaires constructifs de Keith Hobson et Carolyn Callaghan.
\end{abstract}

\section{LITERATURE CITED}

Bijleveld van Lexmond, M., J.-M. Bonmatin, D. Goulson, and D. A. Noome. 2015. Worldwide integrated assessment on systemic pesticides. Environmental Science and Pollution Research 22:1-4. https://doi.org/10.1007/s11356-014-3220-1

Calvert, A. 2012. Research priorities to support the conservation of aerial insectivores in Canada. Bird Studies Canada and Environment Canada, Port Rowan and Ottawa, Ontario, Canada.

Chagnon, M., D. Kreutzweiser, E. A. D. Mitchell, C. A. Morrissey, D. A. Noome, and J. P. Van der Sluijs. 2015. Risks of large-scale use of systemic insecticides to ecosystem functioning and services. Environmental Science and Pollution Research 22:119-134. https://doi.org/10.1007/s11356-014-3277-x

Didham, R. K., F. Barbero, C. M. Collins, M. L. Forister, C. Hassall, S. R. Leather, L. Packer, M. E. Saunders, and A. J. A. Stewart. 2020. Spotlight on insects: trends, threats and conservation challenges. Insect Conservation and Diversity 13:99-102. https://doi.org/10.1111/icad.12409

Furlan, L., A. Pozzebon, C. Duso, N. Simon-Delso, F. SánchezBayo, P. A. Marchand, F. Codato, M. Bijleveld van Lexmond, and J.-M. Bonmatin. 2018. An update of the Worldwide 
Integrated Assessment (WIA) on systemic insecticides. Part 3: alternatives to systemic insecticides. Environmental Science and Pollution Research. https://doi.org/10.1007/s11356-017-1052-5

Gregory, R. D., A. van Strien, P. Vorisek, A. W. G. Meyling, D. G. Noble, R. P. B. Foppen, and D. W. Gibbons. 2005. Developing indicators for European birds. Philosophical Transactions of the Royal Society B 360:269-288. https://doi.org/10.1098/rstb.2004.1602

Hallmann, C. A., R. P. B. Foppen, C. A. M. van Turnhout, H. de Kroon, and E. Jongejans. 2014. Declines in insectivorous birds are associated with high neonicotinoid concentrations. Nature 511:341-343. https://doi.org/10.1038/nature13531

Hallmann C. A., M. Sorg, E. Jongejans, H. Siepel, N. Hofland, H. Schwan, W. Stenmans, A. Müller, H. Sumser, T. Hörren, D. Goulson, and H. de Kroon. 2017. More than 75 percent decline over 27 years in total flying insect biomass in protected areas. PLoS ONE 12:e185809. https://doi.org/10.1371/journal. pone.0185809

Harvey, J. A., R. Heinin, I. Armbrecht, Y. Basset, J. H. BaxterGilbert, T. M. Bezemer, M. Böhm, R. Bommarco, P. A. V. Borges, P. Cardoso, et al. 2020. International scientists formulate a roadmap for insect conservation and recovery. Nature Ecology and Evolution 4:174-176. https://doi.org/10.1038/s41559-019-1079-8

Imlay, T. L., J. Mills Flemming, S. Saldanha, N. T. Wheelwright, and M. L. Leonard. 2018. Breeding phenology and performance for four swallows over 57 years: relationships with temperature and precipitation. Ecosphere 9:e02166. https://doi.org/10.1002/ ecs2.2166

Kleijn, D., R. Bommarco, T. P. M. Fijen, L. A. Garibaldi, S. G. Potts, and W. H. van der Putten. 2019. Ecological intensification: bridging the gap between science and practice. Trends in Ecology \& Evolution 34:154-166. https://doi.org/10.1016/j.tree.2018.11.002

Longcore, T., and P. A. Smith. 2013. On avian mortality associated with human activities. Avian Conservation and Ecology 8(2):1. http://dx.doi.org/10.5751/ACE-00606-080201

Michel, N. L., A. C. Smith, R. G. Clark, C. A. Morrissey, and K. A. Hobson. 2016. Differences in spatial synchrony and interspecific concordance inform guild-level population trends for aerial insectivorous birds. Ecography 39:774-786. https://doi. org/10.1111/ecog.01798

Møller, A. P. 2019. Parallel declines in abundance of insects and insectivorous birds in Denmark over 22 years. Ecology and Evolution 9:6581-6587. https://doi.org/10.1002/ece3.5236

Montgomery, G. A., R. R. Dunn, R. Fox, E. Jongejans, S. R. Leather, M. E. Saunders, C. R. Shortall, M. W. Tingley, and D. L. Wagner. 2020. Is the insect apocalypse upon us? How to find out. Biological Conservation 241:108327. https://doi.org/10.1016/ j.biocon.2019.108327
Nebel, S., A. Mills, J. D. McCracken, and P. D. Taylor. 2010. Declines of aerial insectivores in North America follow a geographic gradient. Avian Conservation and Ecology - Écologie et conservation des oiseaux 5(2):1. https://doi.org/10.5751/ ACE-00391-050201

North American Bird Conservation Initiative (NABCI). 2019. The state of Canada's birds 2019. NABCI Canada, Gatineau, Québec, Canada. [online] URL: http://nabci.net/resources/stateof-canadas-birds-2019/

Rosenberg, K. V., A. M. Dokter, P. J. Blancher, J. R. Sauer, A. C. Smith, P. A. Smith, J. C. Stanton, A. Panjabi, L. Helft, M. Parr, and P. P. Marra. 2019. Decline of the North American avifauna. Science 365:120-124. https://doi.org/10.1126/science.aaw1313

Spiller, K. J., and R. Dettmers. 2019. Evidence for multiple drivers of aerial insectivore declines in North America. Condor 121: duz010. https://doi.org/10.1093/condor/duz010

Tang, J., J. Wice, V. G. Thomas, and P. G. Kevan. 2007. Assessment of Canadian federal and provincial legislation to conserve native and managed pollinators. International Journal of Biodiversity Science and Management 3:46-55. https://doi.org/10.1080/17451590709618161

van Klink, R., D. E. Bowler, K. B. Gongalsky, A. B. Swengel, A. Gentile, J. M. Chase. 2020. Meta-analysis reveals declines in terrestrial but increases in freshwater insect abundances. Science 368:417-420. https://doi.org/10.1126/science.aax9931

Wagner, D. L. 2020. Insect declines in the Anthropocene. Annual Review of Entomology 65:457-480. https://doi.org/10.1146/ annurev-ento-011019-025151

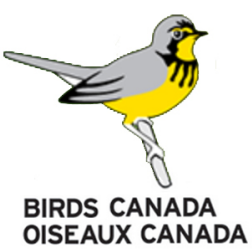

